\title{
A study of variations in formation of superficial palmar arch in Nepalese cadavers
}

\author{
Prakash Baral* (D), Rami Shrestha, Subash Sapkota, Sapana Koju, Binod Chaudhary
}

Department of Anatomy, Gandaki Medical College, Pokhara, Kaski, Nepal

\begin{abstract}
Introduction: The superficial palmar arch (SPA) is the main source of arterial supply to the palm. It is an arterial arcade formed mainly by the ulnar artery and is completed by the superficial palmar branch of the radial artery. The study was conducted with objective to study the variations in formation of superficial palmar arch in Nepalese cadaveric hands. Methodology: Two horizontal incisions were made, one at the wrist joint and another incision along the root of fingers. A vertical incision was made from the middle of the wrist to the 3rd metacarpo-phalangeal joint. The skin of the palm and the palmar aponeurosis was reflected. The surrounding adipose tissue was removed to view the superficial palmar arch and its branches. Result: In present study, out of 50 specimens of hands, $64 \%$ of superficial palmar arch was formed as normal pattern and in $36 \%$ cases it was formed by ulnar artery alone. Normal SPA pattern was seen $63 \%$ in male and $66.7 \%$ in female i.e. higher percentage in female than in male but it was statistically insignificant $(\mathrm{P}<0.05)$. SPA-ulnar artery alone pattern was seen $37 \%$ in male and $33.3 \%$ in female i.e. higher percentage in male than in female, however it was also statistically insignificant $(\mathrm{P}<0.05)$. Conclusion: Normal pattern of SPA observed in Sixty-four percent of Nepalese hands and in thirty-six percent cases it was formed by ulnar artery alone. There was no statistically significant difference in SPA pattern distribution between Nepalese male and female.
\end{abstract}

Keywords: superficial palmar arch, ulnar artery, radial artery

\section{*Correspondence:}

Dr. Prakash Baral

Professor, Department of Anatomy, Gandaki

Medical College, Pokhara, Kaski, Nepal

E-mail: prakashbaral2002@gmail.com

Submitted: January 1, 2021

Accepted: June 13, 2021

To cite: Baral P, Shrestha R, Sapkota S, Koju S, Chaudhary B. A study of variations in formation of superficial palmar arch in Nepalese cadavers. JGMC Nepal. 2021;14(1):19-23.

DOI: $10.3126 /$ jgmcn.v14i1.33995

Contact no: $+977-9804125022$

\section{INTRODUCTION}

The ulnar artery, accompanied by ulnar nerve on its medial side, enters the palm superficial to the flexor retinaculum and on the radial side of pisiform bone. Beneath the Palmaris brevis the artery divides into superficial and deep branches. The superficial branch is the direct continuation of the ulnar artery and forms the main contribution of superficial palmar arch (SPA). Superficial palmar arch is an arterial arcade which lies beneath the palmar aponeurosis and in front of long flexor tendons, lumbrical muscles and palmar digital branches of median nerve. The arch is formed by the superficial terminal branch of ulnar artery and completed on the lateral side by one of the following arteries:

a) Superficial palmar branch of radial artery

b) Arteria princeps pollicis

c) Arteria radialis indicis or

d) Arteria nervi mediana which accompanies the median nerve

The convexity of arch is directed distally on a level with the distal border of outstretched thumb. ${ }^{1}$ 
SPA has been put forth by many researchers. Few to be noted are as follow: Huber ${ }^{2}$ groups the superficial palmar arch into two classes, arches where in participating arteries form a complete arch (show anastomosis) and those that form incomplete arch (fail to anastomose). Gellman ${ }^{3}$ also classified the superficial palmar arch into two classes. It usually communicates with the superficial branch of the radial artery to complete the arch. In incomplete variety of SPA, no anastomosis is seen between the ulnar and radial arteries.

Rationale of study: So far variations in formation of superficial palmar arch in Nepalese cadavers had not been conducted thus this study was undertaken. The findings of present study would contribute to the Nepali data base in regard to variation pattern of superficial palmar arch.

Importance of study: The findings of present study will be useful for anatomists, orthopedic surgeons while doing surgical operations on hand and radiologist while doing angiography in upper limb.

\section{Objectives:}

\section{General objective:}

To study the variations in formation of superficial palmar arch in Nepalese cadaveric hands.

\section{Specific objectives:}

1. To study the variations in formation of superficial palmar arch in Nepalese male cadaveric hands.

2. To study the variations in formation of superficial palmar arch in Nepalese female cadaveric hands.

3. To compare the variations in formation of superficial palmar arch in Nepalese male and female cadaveric hands.

\section{METHODS}

Study Design and study setting: It is the cross-sectional study. This study was carried out from July 2014 to August 2019 in dissection hall of Anatomy, Gandaki Medical College, Pokhara, Nepal.

Sampling method and sample size: The non-probability convenience sampling technique was adopted. All cadaveric hands available in dissection room of anatomy department during five years were studied thus sample size was not calculated as the study followed convenience sampling method.

Procedure: A total number of 50 hands of 38 males and 12 females, from embalmed human cadavers, fixed in $10 \%$ formaldehyde solution were dissected in the department of Anatomy, Gandaki Medial College. Two horizontal incisions were made, one at the wrist joint and another incision along the root of fingers. A vertical incision was made from the middle of the wrist to the 3rd metacarpo-phalangeal joint. The skin of the palm and the palmar aponeurosis was reflected. The surrounding adipose tissue was removed to view the superficial palmar arch and its branches. ${ }^{4}$ The superficial palmar arches were studied, and the variations found were noted down.

Reliability and validity of research: All the findings were observed and noted down by principal author so there was no inter-observer error or bias. As this is anatomy dissection based study it was highly reliable and valid. Adequate sample size, adaptation of proper research methodology, due care given during data collection and entry, correct use of statistical tests etc enhanced the reliability and validity of present study.

Ethical consideration: As present study was carried out on cadaveric (dead bodies) hands there was no ethical issue, but ethical approval was taken from institutional ethical committee, Gandaki Medical College, Kaski, Nepal. The reference number for ethical approval letter is 30-02-071. The letter was issued on 13th June 2014.

Some working definitions for the present study are as follows: "Normal pattern" of SPA arch means the arch formed by the superficial terminal branch of ulnar artery and completed on the lateral side by Superficial palmar branch of radial artery. It is complete form of arch. "Incomplete pattern" of SPA means the arch formed by only one artery on one side. If SPA is formed by the superficial terminal branch of ulnar artery alone then it is "SPA by Ulnar artery alone pattern". However, If SPA is formed by other than the superficial terminal branch of ulnar artery alone then it is "SPA by other artery alone pattern".

Statistical analysis: The data were entered into excel sheet first. The frequency percentage was calculated. The statistical test, i.e. chi -square test was applied to test the significant variation between male and female. The SPSS version 16 was used for the test. For the data quality control during present research study, measures taken were adopting correct method of data collection; screening and verifying the collected data; selecting appropriate computer software; coding and entering the data into computer and recheck it; processing the data; constructing a data base for data management and appropriate data analysis and review over it. 


\section{RESULTS}

A total number of 50 hands of 38 males and 12 females were studied. In this study, normal pattern of superficial palmar arch meant the arch formed by the superficial terminal branch of ulnar artery and completed on the lateral side by Superficial palmar branch of radial artery. It was complete form of arch; one variation type observed during study was superficial palmar arch formed by the superficial terminal branch of ulnar artery alone; another variation type meant the superficial palmar arch formed by other ways mentioned above. It was not observed during study.

Table 1: Variation patterns in formation of superficial palmar arch in cadaveric hands in overall cases

\begin{tabular}{lll}
\hline SN. & Patterns & Overall (n=50 hands) \\
1 & Normal/common & $64 \%(\mathrm{n}=32)$ \\
2 & Ulnar artery alone variant & $36 \%(\mathrm{n}=18)$ \\
3 & Other variants & $0 \%(\mathrm{n}=0)$ \\
\hline
\end{tabular}

Sixty-four percent (64\%) showed normal/common pattern of SPA; $36 \%$ SPA by ulnar artery alone pattern and other variant patterns were not found in the present study.

Table 2: Variation patterns in formation of superficial palmar arch in male cadaveric hands.

\begin{tabular}{lll}
\hline SN. & Pattern & Male $(\mathrm{n}=38$ hands $)$ \\
1 & Normal & $63 \%(\mathrm{n}=24)$ \\
2 & Ulnar artery alone & $37 \%(\mathrm{n}=14)$ \\
3 & Other variants & $0 \%(\mathrm{n}=0)$ \\
\hline
\end{tabular}

Sixty-three percent (63\%) showed normal pattern of SPA in male hands; $37 \%$ SPA by ulnar artery alone pattern and others pattern was not found in the present study.

Table 3: Variation patterns in formation of superficial palmar arch in female cadaveric hands

\begin{tabular}{lll}
\hline SN. & Pattern & Female $(\mathrm{n}=12$ hands) \\
1 & Normal & $66.7 \%(\mathrm{n}=8)$ \\
2 & Ulnar artery alone & $33.3 \%(\mathrm{n}=4)$ \\
3 & Other variants & $0 \%(\mathrm{n}=0)$ \\
\hline
\end{tabular}

Of total, $66.7 \%$ showed normal pattern of SPA in female hands; 33.3\% SPA by ulnar artery alone pattern and others pattern was not found in the present study.
Table 4: Comparison of variation patterns in formation of superficial palmar arch between male and female cadaveric hands

\begin{tabular}{lllll}
\hline SN. & Pattern & Male $(\mathrm{n}=38$ hands) & Female $(\mathrm{n}=12$ hands) & P value \\
1 & Normal & $63(\mathrm{n}=24)$ & $66.7 \%(\mathrm{n}=8)$ & 0.06 \\
2 & Ulnar artery alone & $37 \%(\mathrm{n}=14)$ & $33.3 \%(\mathrm{n}=4)$ & 0.06 \\
3 & Other variants & $0 \%(\mathrm{n}=0)$ & $0 \%(\mathrm{n}=0)$ & \\
\hline
\end{tabular}

(Statistical significance, $\mathrm{P}<0.05$ )

Though normal SPA pattern was seen $63 \%$ in male and $66.7 \%$ in female i.e. higher percentage in female than in male, it was statistically insignificant $(\mathrm{P}<0.05)$. However, SPA-ulnar artery alone pattern was seen $37 \%$ in male and $33.3 \%$ in female i.e. higher percentage in male than in female but it was statistically insignificant $(\mathrm{P}<0.05)$.

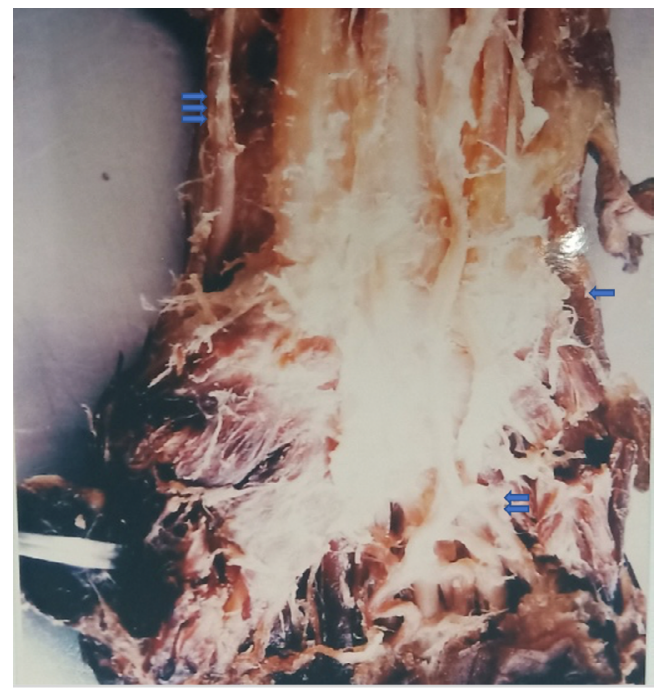

Figure 1: Superficial palmar arch formed by ulnar artery alone. Ulnar Artery (single arrow), superficial palmar arch (double arrow), radial artery (triple arrow)

\section{DISCUSSION}

In present study, out of 50 specimens (hands), 36\% of superficial palmar arch was formed by ulnar artery alone and $64 \%$ was formed by normal/common pattern.

A study by Gupta et.al ${ }^{5}$ on a morphological study of variations in superficial palmar arches of the hand found that in $77.3 \%$ of specimens superficial palmar arch was complete and in $22.6 \%$ of specimens it was incomplete which is similar to present study.

Similarly, Joshi et.al ${ }^{6}$ also found that $82 \%$ showed complete superficial palmar arches and a very low incidence (18\%) of incomplete arches. Jose et.al ${ }^{4}$ also found that $96 \%$ of SPA were complete and $4 \%$ incomplete. Ranjan et.al ${ }^{7}$ also 
found that complete superficial palmar arch variations were observed in 111 cadaveric hands; whereas there were 29 cadaveric hand with the variations incomplete superficial palmar arch. Similarly, a complete superficial arch was observed by Mbaka et.al ${ }^{8}$ in $73.9 \%$ specimens and incomplete arch constituted $26.1 \%$ of the entire palmar arch formation.

A study on Palmar Arterial Arches- A Morphological Study by Patnaik et.al ${ }^{9}$ found that superficial palmar arch was single in $94 \%$ limbs (complete in $78 \%$, \& incomplete in $16 \%$.) and in $6 \%$ of limbs, a double Superficial palmar arch was found, proximal complete (radio ulnar in $4 \%$ \& mediano-ulnar in 2\%) \& distal incomplete.

A study conducted by Salgado et.a ${ }^{10}$ revealed that specimens with contribution from both the radial and the ulnar arteries for the superficial palmar arches were $80 \%$, while specimens with the contribution solely from the ulnar artery were $12 \%$ which is similar to our study.

A case study by Raj et.al ${ }^{11}$ showed that there was incomplete superficial palmar arch on right side which was not completed by any of the branches of radial or median artery. On the left side of the same individual, the superficial palmar arch was classical, completed by superficial branch of radial artery.

Normal SPA pattern was seen $63 \%$ in male and $66.7 \%$ in female i.e. higher percentage in female than in male but it was statistically insignificant $(\mathrm{P}<0.05)$. SPA-ulnar artery alone pattern was seen $37 \%$ in male and $33.3 \%$ in female i.e. higher percentage in male than in female, however it was also statistically insignificant $(\mathrm{P}<0.05)$.

Like the present study, no other study made comparison between male and female.

\section{Embryological basis of the finding}

There are very few schools of thought which explain the development of the arterial pattern in humans. As suggested by De Vriese, ${ }^{12}$ the arteries arise from the initial capillary reticulum in association with each of the principal nerves. Singer opined the development of the arterial system by a bud formation from the axial arterial trunk. ${ }^{13}$ A study which was conducted by Rodriguez- Niedenfuhr on human embryos, described the commencement of the vascular system at stage 12 (3-5mm Crown-rump length; 26th day) and differentiation of the arterial wall into all the segments, which included the radial artery and the palmar arch in stage 23 (27-31mm Crown-rump length; 54th day). According to this theory, a capillary plexus is formed, which enters the limb bud and grows along with it distally. Thereafter, only the axial artery supplies the limb and the capillary plexus, giving rise to the arteries of the antebrachial region through a sprouting mechanism. ${ }^{14}$

The sprouting may join with the developing ulnar artery only, instead of joining to both ulnar and radial arteries, and form the superficial palmar arch during the developmental process as in our finding.

\section{Limitations of study:}

Study was conducted on cadaveric hands which were available in less number thus the sample size was less in number. The similar study should be carried out in other regions of Nepal and meta-analytical study should be done in future.

\section{CONCLUSION}

Sixty-four percent showed normal pattern of SPA; 36\% SPA by ulnar artery alone pattern and others pattern was not found in present study. Normal SPA pattern was seen $63 \%$ in male and $66.7 \%$ in female i.e. higher percentage in female than in male but it was statistically insignificant. SPA-ulnar artery alone pattern was seen $37 \%$ in male and $33.3 \%$ in female i.e. higher percentage in male than in female, however it was also statistically insignificant.

Conflict of interest: None declared,

\section{REFERENCES}

1. Datta AK. Essentials of human anatomy, superior and inferior extremities. 4th ed. Kolkata: Current books international; 2010.

2. Huber GC. Piersol's Human Anatomy: the vascular system. 9th ed. Philadelphia: JB Lippincott Co; 1930.

3. Gellman H, Botte MJ, Shankwiler J, Gelberman RH. Arterial patterns of the deep and superficial palmar arches. Clin Orthop Relat Res. 2001;383E:41-6. DOI: 10.1097/00003086200102000-00007

4. Jose BA, Rekha S, Babu S. Analysis of the vascular pattern in the superficial palmar arch formation. Indian Journal of Clinical Anatomy and Physiology. 2017;4(1):11-5.

5. Gupta C, Kalthur SG, Nair N, Pai SR. A morphological study of variations in superficial palmar arches of the hand. Journal of Health and Research. 2015;2(2):140-4. DOI: 10.4103/23483334.153259

6. Joshi SB, Vatsalaswamy P, Bahetee BH. Variation in Formation of Superficial Palmar Arches with Clinical Implications. Journal of Clinical and Diagnostic Research. 2014;8(4):6-9. DOI: $10.7860 / J C D R / 2014 / 7078.4252$ 
7. Ranjan R, Dubey AK, Jain A. Supercial Palmar Arch: a study of morphological Variation patterns. Global journal for research analysis. 2017;6(5):621-3

8. Mbaka GO, Ejiwunmi AO, Olabiyi OO. Pattern of variations in superficial palmar arch in 134 Negro cadaveric hands. Italian journal of anatomy and embryology. 2014;119(3):153-62

9. Patnaik VVG, Kalsey G, Singla RK. Palmar Arterial Arches- A Morphological Study. J Anat. Soc. India. 2002;51(2):187-93

10. Salgado LSS, Hasan R, Perera AAMMSL, Wijesundara WMRD, Anuradha WK. Variations of the Superficial Palmar Arch in a Sri Lankan Perspective: A Cadaveric Study. Journal of Advances in Medical Research. 2014;1(2):61-7. DOI: 10.5176/2345-7201_1.2.25

11. Raj RP, More AB, Kunjumon PC. Unilateral incomplete superficial palmar arch variation. Int. J. Bioassays. 2014;3(1):1692-4

12. DeVrieseB, RecherchessurL.Evolutiondesvaisseauxsanguins des members chez l' home. Archiv Biol. 1902;18:665-730. DOI: 10.3406/ccmaa.1902.11321

13. Singer E. Embryological pattern persisting in the arteries of the arm. Anatomical Record. 1933;55:403-9. DOI: 10.1002/ ar.1090550407

14. Rodriguez-Niedenfuhr M, Burton GJ, Deu J, Sanudo JR. Development of the arterial pattern in the upper limb of staged human embryos: normal development and anatomic ariations. J Anat. 2001;199:407-17. DOI: 10.1046/j.14697580.2001.19940407.x 